\title{
Temporal brightness enhancement: Studies of individual differences
}

\author{
RICHARD W. BOWEN \\ Loyola University of Chicago, Chicago, Illinois
}

\begin{abstract}
We have previously identified categorical individual differences in the occurrence of temporal brightness enhancement (TBE) by using a simultaneous brightness discrimination paradigm (Bowen \& Markell, 1980). TBE is a nonmonotonic relation between brightness and pulse duration, pulses of intermediate duration (75-125 msec) can appear brighter than longer or shorter pulses of the same luminance. Three classes of observers can be defined based on whether they perceive TBE under one of two conditions of temporal asynchrony between a short test pulse and a longer (500 msec) comparison pulse: simultaneous onset of the pulses or simultaneous offset. Type A observers show TBE for both asynchrony conditions; Type B observers show the effect for simultaneous offset but not simultaneous onset; Type $\mathrm{C}$ observers do not show TBE for either asynchrony. In the present study, we show that Type $\mathrm{A}$ and Type $\mathrm{C}$ observers maintain a constant brightnessduration relation as the asynchrony between test and comparison pulses is varied from simultaneous onset to simultaneous offset. Type B observers show a gradual shift in the brightnessduration relation as asynchrony changes. In a separate experiment, we find that practice has little effect on Type A and Type B observers but that Type C observers may change in classification to Types A and B over as few as five experimental sessions. The hypothesis that individual differences are due to differential "weighting" of chromatic (sustained) and achromatic (transient) visual channels is discussed.
\end{abstract}

Implicit in the design of psychophysical investigations of sensory function is the expectation that obtained results will be consistent for different observers. Observers who behave similarly usually increase one's confidence that the behavioral task at hand is tapping a basic aspect of sensory processing. Individual differences, especially qualitative differences, among observers may therefore go unreported. We have recently been investigating an interesting pattern of individual differences involving an effect in visual perception called temporal brightness enhancement (TBE). Frequently investigated since its discovery (Broca \& Sulzer, 1902), TBE is indicated by a nonmonotonic relation between the brightness of a pulse of light and the duration of the pulse. Specifically, very short or long pulses do not seem as bright as intermediateduration pulses $(50-150 \mathrm{msec})$. We have found that TBE does not occur for some observers and that among other observers the effect may be manifest under certain stimulus conditions but not others (Bowen \& Markell, 1980).

We have been using a particular simultaneous brightness discrimination paradigm to measure the brightnessduration relation (Bowen \& Pokorny, 1978). Observers compare the brightness of a short (test) pulse and a long (comparison) pulse of equal luminance. The pulses are presented under two temporal asynchrony conditions,

Supported by National Science Foundation Grant BNS 81-11366 to R. W. Bowen. I thank Dr. Joel Pokorny for a critical reading of the manuscript.

The author's mailing address is: Department of Psychology, Loyola University of Chicago, Chicago, IL 60626. simultaneous onset of pulses or simultaneous offset. Three classes of observers can be defined: Type A observers show TBE under both asynchrony conditions; they represent $48 \%$ of the sample of 220 observers tested to date. Type B observers ( $38 \%$ of the sample) show TBE for simultaneous offset of pulses but not for simultaneous onset. The remaining, Type C, observers (14\% of the sample) give no evidence of TBE for either asynchrony condition. These individual differences are reliably observed and have been noted in other studies of TBE (Osaka, 1982).

These classes of observers have been tested on other measures of visual performance: sine-wave flicker modulation sensitivity, contrast sensitivity for moving sine-wave gratings, and magnitude estimation of the brightness of single light pulses (Bowen, Sekuler, Owsley, \& Markell, 1982). The three types of observers gave similar results for flicker and motion sensitivity. This suggested that individual differences are not due to general differences in visual temporal processing or to differences in underlying visual neurophysiology. But the types of observer did differ in magnitude estimation measures of the brightnessduration relations. Type A observers showed TBE, but Type C observers did not. Half of the Type B observers gave evidence of brightness enhancement. This suggested that individual differences are related to differing perceptual criteria used in judging the brightness of isolated pulses.

The present study was designed to address two empirical issues concerning these individual differences. The first issue (Experiments 1 and 2) was whether individual 
differences would be evident for a range of temporal asynchronies between test and comparison pulses. From previous work, we knew that in Type A observers TBE is present and with Type $\mathrm{C}$ is absent, independent of whether the pulses come on together or go off together. For Type B observers, changing the temporal asynchrony produces a differential effect. Type B observers must shift from nonoccurrence to occurrence of TBE as asynchrony is varied from simultaneous onset to simultaneous offset. Would this shift be gradual or abrupt, and would Type A and Type $\mathrm{C}$ observers perform any differently at intermediate pulse asynchronies?

The second issue (Experiment 3) was the stability of individual differences, - whether the classification of an observer as A, B, or C could be reliably measured over a number of practice sessions.

\section{EXPERIMENTS 1 AND 2}

\section{Method}

Observers. A total of 58 undergraduates fulfilling a requirement of a general psychology course participated in the study. Twenty-five observers were used in Experiment 1;33 were used in Experiment 2. All observers were screened for stereopsis and for visual acuity of $6 / 6$ or better (with or without corrective lenses) with a Bausch and Lomb orthorator.

Psychophysical method. The method we employed to measure brightness vs. duration relations was the following: (Bowen \& Markell, 1980; Bowen \& Nissen, 1979; Bowen \& Pokorny, 1978; Bowen et al., 1982). On each trial, the observer was presented with two equal-luminance pulses of light seen in haploscopic view. One pulse-the comparison pulse-was $500 \mathrm{msec}$ in duration. The shorter pulse varied in duration from 20 to $320 \mathrm{msec}$, spanning the normal temporal range of the TBE effect. The long and short pulses were presented at one of several temporal asynchronies. The observer reported which pulse ("left" or "right") appeared brighter, but was not specifically advised that a 500msec comparison pulse would be presented on each trial. Over trials, the duration of the shorter pulse, its location, and the stimulus onset asynchrony (SOA) between pulses were randomly varied.

Data from this procedure are functions relating the percentage of trials on which the longer comparison pulse was judged brighter to the duration of the shorter pulse. The occurrence of TBE is indicated by a dip in the function below 50\%: some short pulse is consistently judged brighter than the 500-msec comparison pulse. If TBE does not occur, this function should be significantly above $50 \%$ for very short test pulses (brightness increases with duration) and near 50\% for long test pulses (brightness does not vary with duration).

Apparatus and Procedure. The apparatus (Bowen \& Pokorny, 1978) was a four-channel optical projection system using a $150-\mathrm{W}$ tungsten halogen lamp as a source. The stimulus array, imaged on rear-projection screens, consisted of two circular targets ( $34^{\prime}$ diam) presented separately to the left and right eyes of the observer. The targets were separated by $2^{\circ} 50^{\prime}$ in haploscopic view. A circular fixation target (13' diam) was positioned between the targets and was fused binocularly by the observer. The pulsed tagets had a luminance of $100 \mathrm{~cd} / \mathrm{m}^{2}$. The array was viewed in darkness at a distance of $0.4 \mathrm{~m}$.

Five test pulse durations were used: $20,40,80,160$, and $320 \mathrm{msec}$. In Experiment 1, four SOA conditions were used: $0 \mathrm{msec}$ (simultaneous onset of test and comparison pulses), $100 \mathrm{msec}$ (onset of test pulse delayed by $100 \mathrm{msec}$ ), $250 \mathrm{msec}$, and simultaneous offset of test and comparison pulses. In Experiment 2, two additional SOA conditions were used: $-200 \mathrm{msec}$ (onset of test pulse $200 \mathrm{msec}$ prior to the onset of the comparison pulse) and $500 \mathrm{msec}$ (onset of test pulse at offset of the comparison pulse). The complete set of SOA conditions is represented in Figure 1 for a test pulse of $80 \mathrm{msec}$.

Each observer was tested in a single 1-h experimental session. All

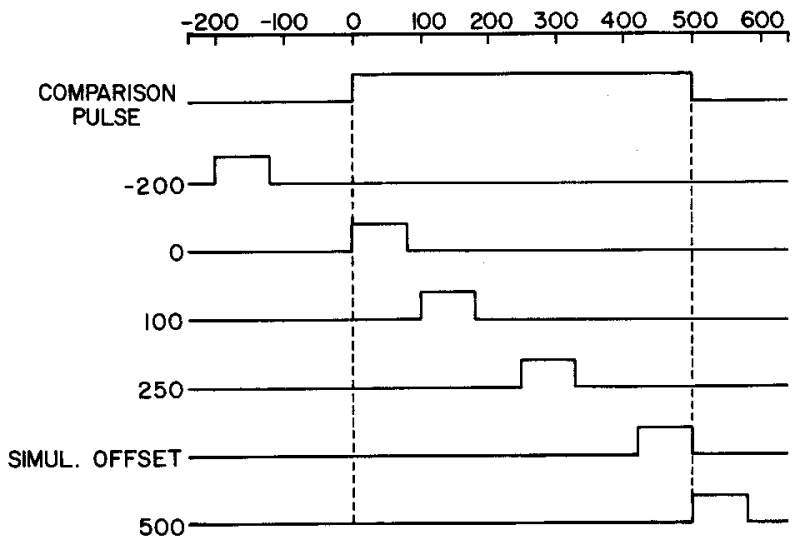

Figure 1. Diagram of the temporal asynchrony conditions used in Experiments 1 and 2, for a test pulse of $80 \mathrm{msec}$. The onset of the comparison pulse has an SOA value of $0 \mathrm{msec}$.

combinations of test-pulse duration, SOA condition, and position of the shorter pulse (left or right) were presented in a randomized block. Ten successive randomized blocks made up the experimental session so that a total of 20 trials ( 10 blocks $\times 2$ positions) were run for each combination of test pulse duration and SOA. In Experiment 1, each observer had 400 trials $(20 \times 5$ pulse durations $\times 4$ SOA values $)$; in Experiment 2,600 trials were run $(20 \times 5$ pulse durations $\times 6$ SOA values $)$. In both experiments, observers were given a 5-min break halfway through the trial sequence.

The experiments were controlled on-line with a microcomputer. The timing of pulse durations and SOA values was accomplished with precision programmable interval timers in the computer mainframe. The observer pushed buttons to initiate trials and signal responses.

\section{Results and Discussion}

The data from Experiment 1 are given in Figure 2, which shows the mean percent of trials the longer pulse was judged brighter as a function of the duration of the shorter pulse. Functions at the four temporal asynchronies (see inset legend) are plotted for the three types of observers. Observers were defined as A, B, or C based only on performance for SOA $=0$ and simultaneous offset. As in previous studies, we indexed TBE by a binomial criterion: $30 \%$ or less of "longer pulse judged brighter" at any pulse duration was taken as evidence of brightness enhancement, since 6 trials out of 20 is significantly lower $(p<.057)$ than $50 \%$ for a binomial distribution, assuming a probability of .5 for responding "longer pulse brighter." From our sample of 33 observers, 10 were Type A, 12 were Type $B$, and 3 were Type $C$.

For Type A observers (left panel), functions at all SOA values show robust TBE effects with a minimum (indicating maximum brightness) at $80 \mathrm{msec}$. The SOA variable produces some separation of the functions, particularly in comparing the function for SOA $=0$ with that for simultaneous offset. This difference in the level but not the shape of these functions was evident in previous studies (Bowen \& Markell, 1980; Bowen et al., 1982), and we discuss the possible significance of this later on.

For Type C observers (right panel), the functions lie between $70 \%$ and $90 \%$ for a test pulse of $20 \mathrm{msec}$, and 


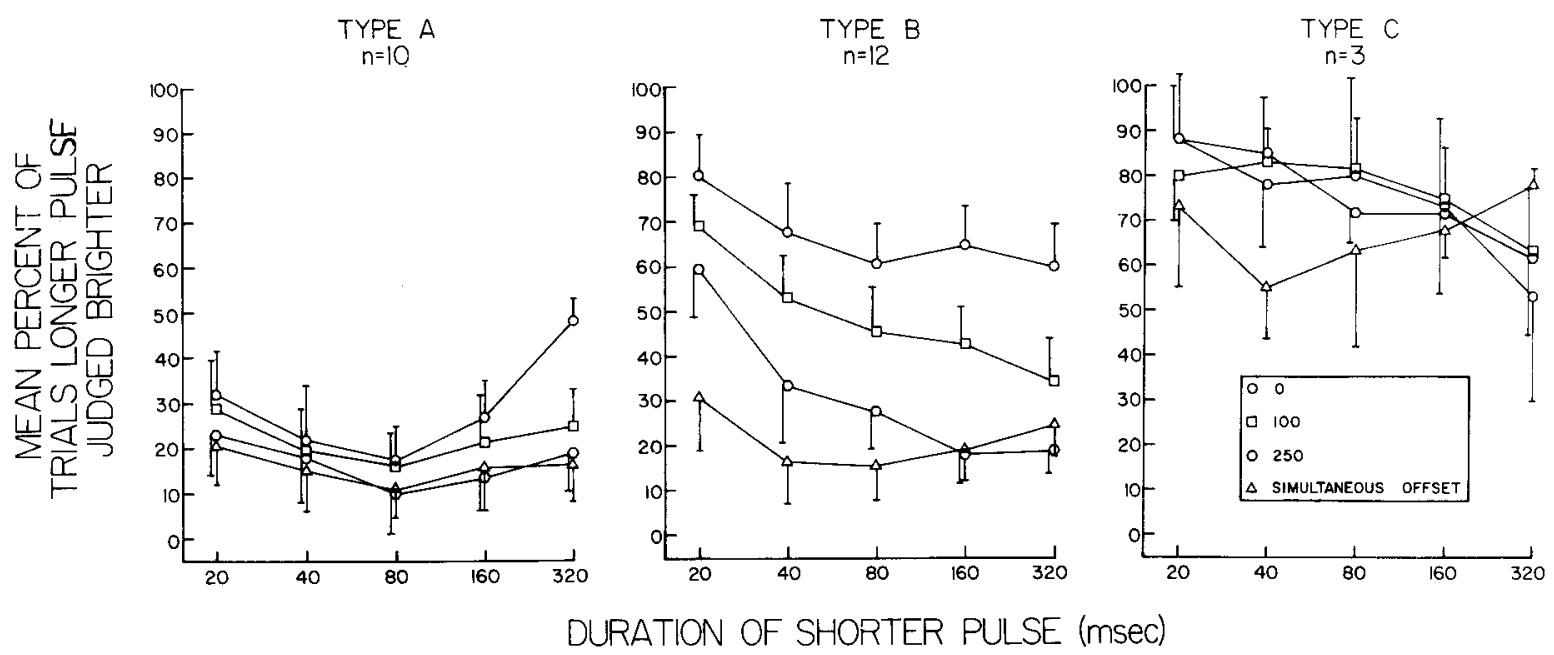

Figure 2. Data from Experiment 1 given as plots of the mean percent of trials on which the longer comparison pulse was judged brighter as a function of the duration of the shorter pulse for Type A (left panel), Type B (middle panel), and Type C (right panel) observers. The inset identifies SOA parameters for the functions. The error bars in this and subsequent figures represent the $95 \%$ confidence intervals for the means (1.96 standard errors).

decrease with duration to levels somewhat above $50 \%$. Thus, for all SOA values, brightness increases (relative to the 500 msec comparison pulse) with increasing test pulse duration, but there is no evidence of TBE. There is also no systematic separation of the functions at different SOAs.

For Type B observers (middle panel), there is a progressive downward displacement of the function going from SOA $=0$ to simultaneous offset, and a systematic change in the shape of the function. As the onset of the test pulse is slid backward from the onset of the comparison pulse, there is a continuous transition from nonoccurrence to occurrence of TBE.
Figure 3 gives comparable data from Experiment 2. The open symbols are as for Figure 2. The filled circles represent the two additional SOA values tested in Experiment 2, $-200 \mathrm{msec}$ (circles) and $+500 \mathrm{msec}$ (squares). The subject sample included 15 Type A, 13 Type B, and 5 Type $\mathrm{C}$ observers, defined as discussed above.

With SOA values of 0,100 , and $250 \mathrm{msec}$, and simultaneous offset, we closely replicated the results of Experiment 1 using a different group of observers.

At an SOA of $500 \mathrm{msec}$ (onset of test pulse at offset of comparison pulse), the data are comparable to those obtained for the simultaneous offset condition for all three types of observer. At SOA $=-200 \mathrm{msec}$, the function

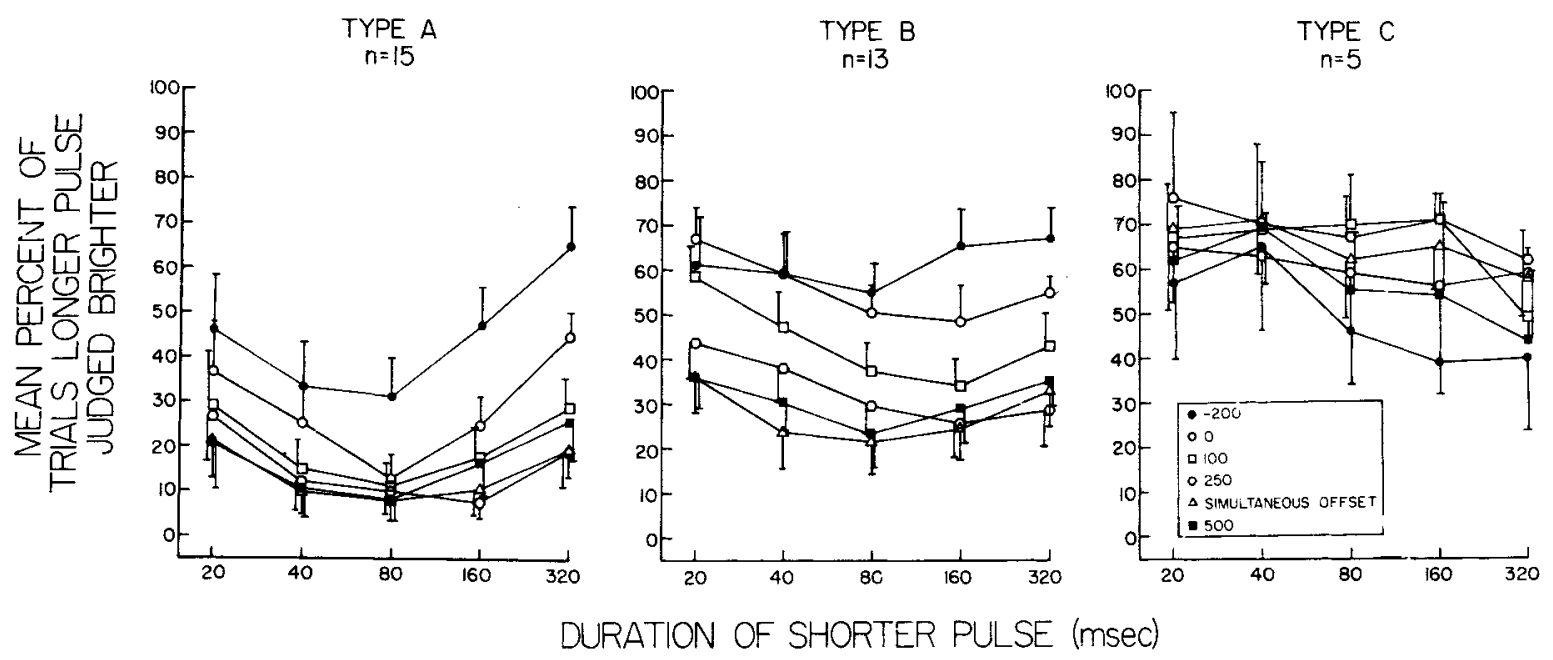

Figure 3. Data from Experiment 2 plotted in the same format as in Figure 2. Open symbols represent SOA values also studied in Experiment 1; filled symbols represent additional values studied in Experiment 3. See inset. The data were obtained from a different sample of observers from those used for Figure 2. 

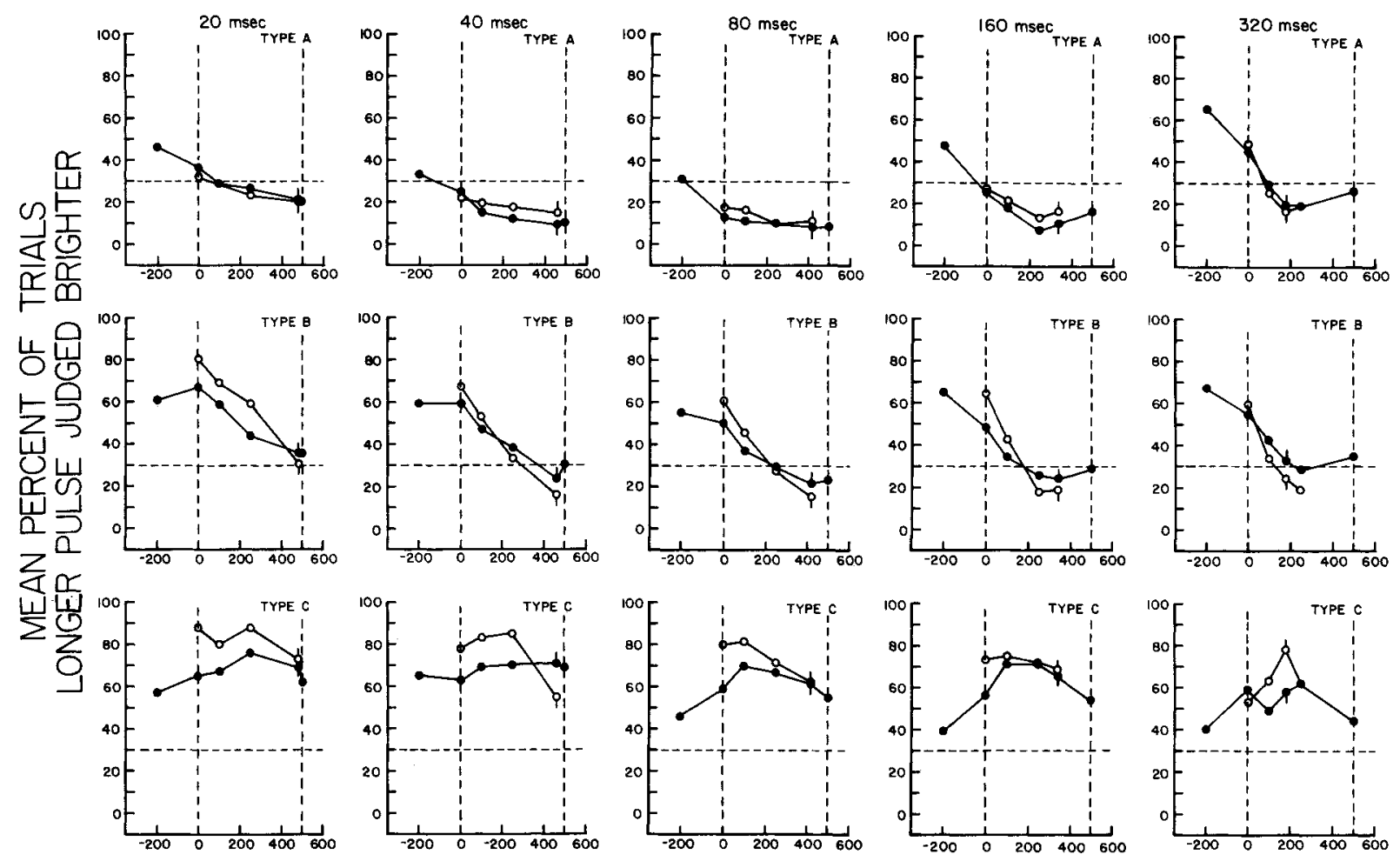

\section{STIMULUS ONSET ASYNCHRONY (msec)}

Figure 4. Data of Figures 2 and 3 replotted as a function of SOA value for each of the five short pulse durations (panels left to right) for each of the observer types (panels top to bottom). Horizontal dashed line marks the binomial criterion level for the occurrence of TBE; vertical dashed lines mark onset and offset of the longer comparison pulse.

for Type A and Type B observers is elevated relative to that for other SOA values and there is a dip in the functions for a test pulse of $80 \mathrm{msec}$. For neither Type A nor Type B observers does the minimum value reach $30 \%$, the criterion percentage for TBE.

The data of Figures 2 and 3 can be replotted to emphasize the dependence of brightness judgments on stimulus onset asynchrony. This is done in Figure 4, which shows mean data as a function of SOA for each of the test pulse durations. Open circles represent the data of Experiment 1 , filled circles, the data of Experiment 2. In each panel, two of the data points have a short vertical line segment pointing up or down. These are data for the simultaneous offset condition, plotted at ihe actual onset asynchrony for the short pulse relative to the onset of the 500 -msec comparison pulse at $\mathrm{SOA}=0$. Vertical dashed lines mark the onset and offset of the comparison pulse; the horizontal dashed line marks the binomial criterion level (30\%) for the occurrence of brightness enhancement.

As noted before, Experiments 1 and 2 produce very similar mean data, since open and filled circles in Figure 3 agree very well. It is also clear that there is a distinct variation in the percentage of trials in which the comparison pulse is judged brighter as a function of the SOA. This variation can be viewed either as a change with asynchrony in the judged brightness of the shorter pulse or as a change in the judged brightness of the comparison pulse. The first case would imply that the brightness of the shorter pulse was not constant, but was modified due, for example, to some spatiotemporal interaction with the comparison pulse at different SOA values. This does not seem a likely possibility, since the pulses are separated by nearly $3^{\circ}$ in haploscopic view, conditions selected to minimize interactions (Bowen \& Pokorny, 1978).

The other possibility, that these data reflect changes in the brightness of the comparison pulse as a function of time, seems more likely. It is assumed that the brightness judgment is made at the time of the onset of the shorter pulse, and we consider data only for SOAs of zero or longer. Given this, the data indicate that for Type A and Type $\mathrm{B}$ observers, the comparison pulse is brightest at $\mathrm{SOA}=$ zero, and declines in brightness at longer SOAs. This response "waveform" is consistent with estimates of the variation over time in the visual response to a pulse of light, based upon a derived impulse response function (Kelly \& Savoie, 1978) or upon reaction time measures to combinations of threshold and suprathreshold luminance gratings (Lupp, Hauske, \& Wolf, 1978). For Type C observers, the brightness of the comparison pulse does not show an initial peak, as if they were not responding to transient features of the comparison stimulus. (See General Discussion.) 
The data at SOA $=-200 \mathrm{msec}$ are a special case, since, for four of the five pulse durations, the brightness judgment is a successive one (there is a temporal gap between test and comparison pulses). As noted above, the data for Type A and Type B observers are elevated at SOA $=-200 \mathrm{msec}$. This can be viewed as a "time order" error (Woodworth \& Schlosberg, 1954): By the time of the onset of the comparison pulse, some representation of the brightness of the test pulse has "decayed" from its initial value.

\section{EXPERIMENT 3}

\section{Method}

Observers. A pool of 36 undergraduates was sampled to obtain eight each of Type A, B, and C observers, based on the binomial criterion cited above. Identification of observers took place during the first of five experimental sessions (see below). Visual screening was as for Experiments 1 and 2 .

Procedure. The apparatus and general psychophysical method were the same as those in Experiments 1 and 2. A total of five experimental sessions were conducted for each observer. The sessions were separated by approximately 1 week. In a given session, five pulse durations $(20,40,80,160$, and $320 \mathrm{msec})$ were tested at three temporal asynchronies, 0 and $100 \mathrm{msec}$ and simultaneous offset. Twenty trials were given at each pulse duration/asynchrony condition with the same protocol as in Experiments 1 and 2. The design of the experiment was sim- ply to give each observer five sessions of practice with no feedback or comment on their performance.

\section{Results}

The results of Experiment 3 are summarized in Figures 5, 6 and 7, which present brightness-duration relations over sessions for Type A, B, and C observers, respectively. The functions have the same format as Figures 2 and 3 and are given for five sessions (panels left to right) and the three SOA values (panels top to bottom).

For Type A (Figure 5) and Type B (Figure 6) observers, the mean data show no systematic changes across sessions at any asynchrony. There appears to be good stability in data collected over a period of at least 4 weeks.

For Type $\mathrm{C}$ observers (Figure 7), there is no obvious change over sessions in the functions at SOA $=0$ and $100 \mathrm{msec}$. For the simultaneous offset condition, however, it appears that a minimum (at $40-80 \mathrm{msec}$ ) develops in the function over the five sessions. Inspection of the individual data for the Type $\mathrm{C}$ observers showed that, for the simultaneous offset condition, three of the eight observers changed from nonoccurrence to occurrence of TBE from the second session on, and five of eight changed by the fifth session. At SOAs of 0 and $100 \mathrm{msec}$, Type C observers tended to alternate between occurrence

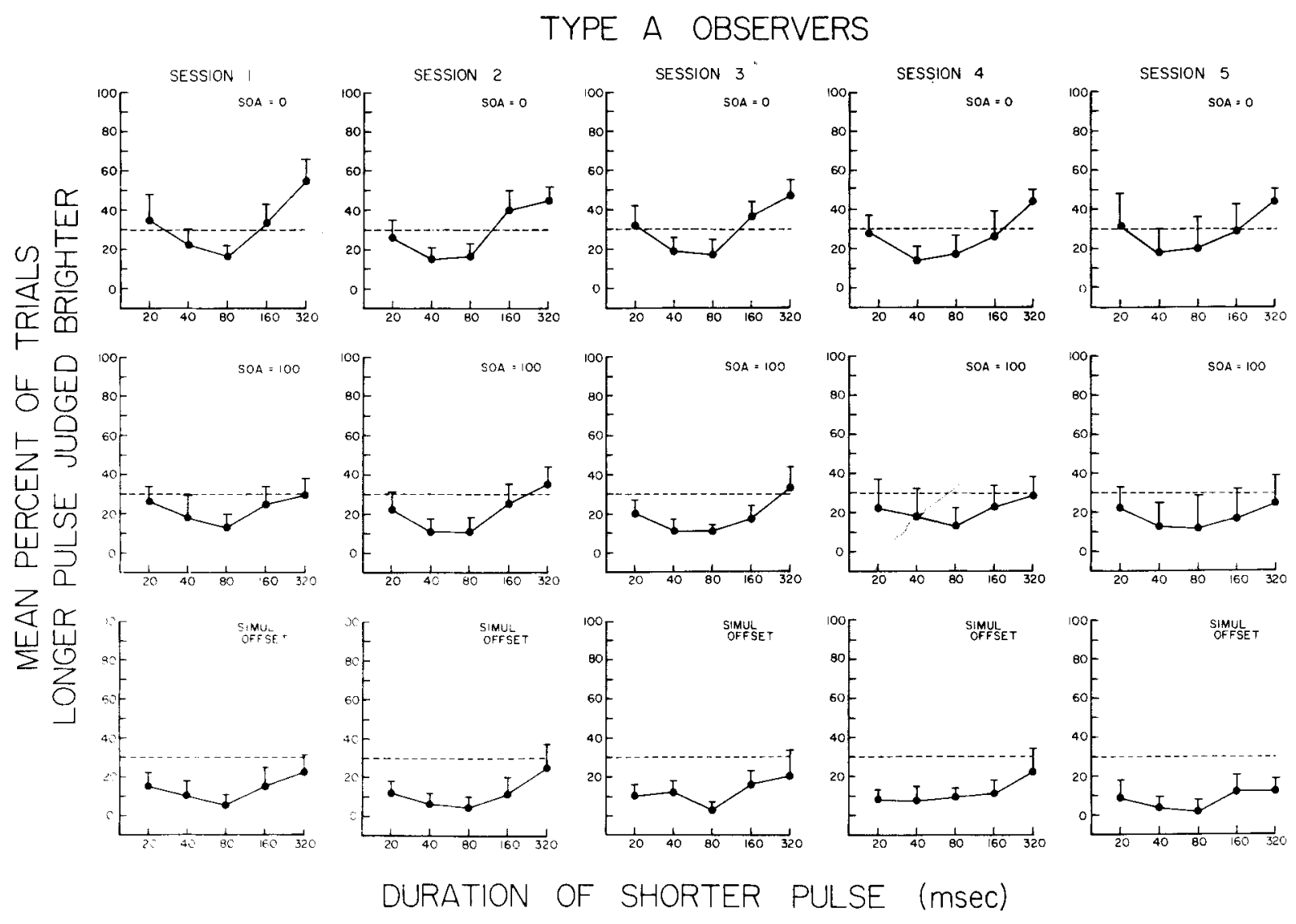

Figure 5. Data from Experiment 3 given as plots of the percent of trials the longer comparison pulse was judged brighter as a function of the duration of the shorter pulse for Type A observers only. Panels left to right represent Practice Sessions 1-5. Panels top to bottom represent SOA values of 0 and 100 msec and simultaneous offset. 


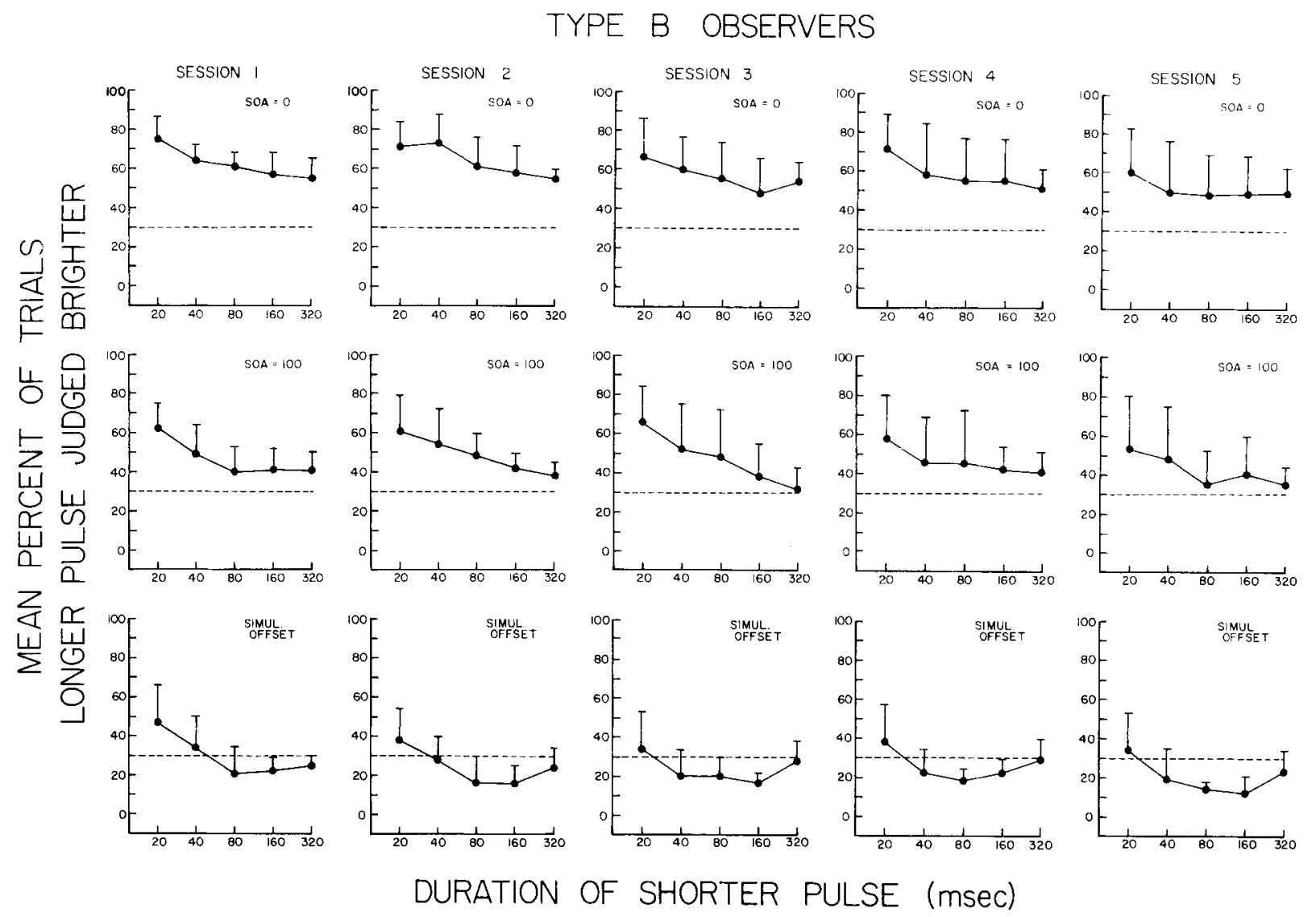

Figure 6. Data for Type B observers in Experiment 3, plotted as for Figure 5.

and nonoccurrence of TBE. This was also true of half of the Type B observers at these SOA values. By contrast, only one of the eight Type A observers showed any change in the effect at any SOA value.

To summarize the results of Experiment 3; Type A observers are very stable in their performance on this paradigm; Type B observers show variability, but tend not to change in type over sessions; for Type C observers. practice with the task produces an increased incidence of TBE. After five sessions, of the eight initial Type C observers, three could be classified as Type B and one as Type A.

\section{GENERAL DISCUSSION}

Based on our previous results with individual differences (Bowen \& Markell, 1980; Bowen et al. 1982), we have suggested that observers may differ in the "perceptual criteria" they adopt in making pulse brightness judgments. The present results do not contradict this explanation. In Experiments 1 and 2, Type $A$ and Type $C$ observers maintain a constant "criterion" at different temporal asynchronies, whereas Type B observers show a transition over temporal asynchrony from a Type $\mathrm{C}$ to a Type A "strategy." Experiment 3 suggests that the criterion adopted by an observer is labile and may change with practice.

As an example of the "perceptual criteria" hypothesis, consider possible strategies in judging the brightness of a prototypical visual response. We assume that the response to a pulse of light increases to a maximum following stimulus onset and then declines to a sustained level following the peak (e.g., Kelly and Savoie, 1978; Lupp et al., 1978). The judged brightness of such a response could involve one of two complementary processes: temporal resolution or temporal integration. Type A observers might adopt the strategy of "resolution" which would assess the peak of the response, whereas Type C observers might "integrate" the response over an appreciable interval. If the size of the response peak for different pulse durations varies nonmonotonically, with a maximum at some intermediate duration (i.e., if TBE is related to some nonlinearity associated with the response peak), then the Type A "resolvers" will show TBE and the Type C "integrators" will not. Type B observers shift between integration and resolution strategies.

A hypothesis of this type is largely ad hoc and we have pointed out previously that even if one settles on a plausible form for the underlying visual response to a pulse (such as that cited immediately above), a variety of pos- 


\section{TYPE C OBSERVERS}
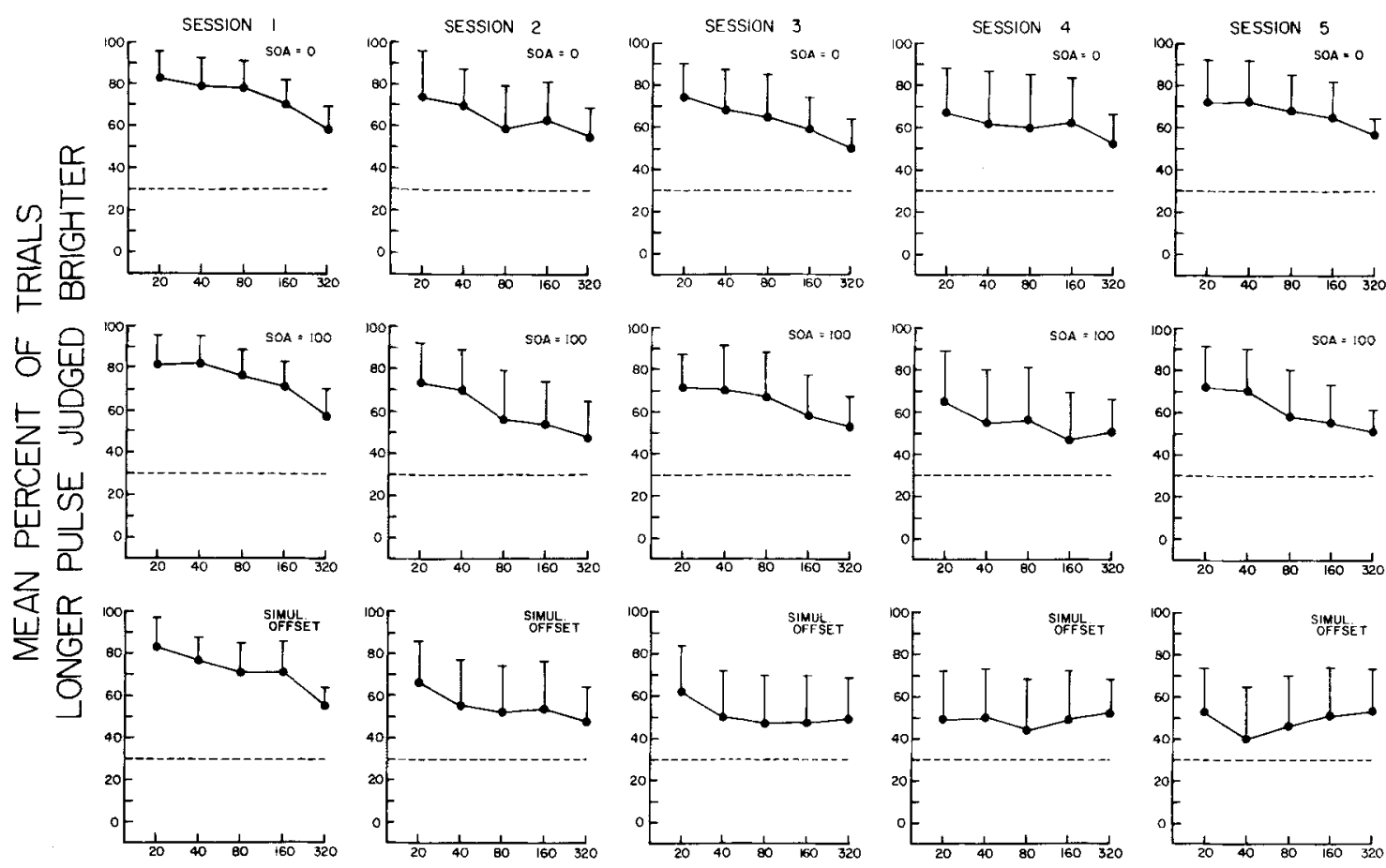

DURATION OF SHORTER PULSE (msec)

Figure 7. Data for Type $\mathrm{C}$ observers in Experiment 3, plotted as for Figure 5.

sible "criteria" involving many possible "features" of the response can be considered. Thus, the metaphor of "resolvers" versus "integrators" will account for the data in a superficial way, but it is one of many possible such hypotheses, and in any event will not itself account for the phenomenon of TBE. ${ }^{1}$

Several psychophysical results concerning the nature of TBE itself point to a more plausible (and testable) hypothesis to account for individual differences. One is the observation that temporal contrast enhancement (enhancement of the apparent contrast of a pulsed grating) is abolished if the grating is "ramped" on over $20 \mathrm{msec}$ rather than being presented as a rectangular pulse (Kitterle \& Corwin, 1983). In addition, Kitterle and Toney (1983) have reported that ramping on a luminance decrement likewise abolishes "temporal darkness enhancement." These results suggest that TBE is associated with a mechanism responding to onset transients. ${ }^{2} \mathrm{~A}$ large body of psychophysical data implies the existence of a "transient" or "phasic" visual mechanism responding to fast temporal transients that is distinct from a "sustained" or "tonic" visual mechanism with a sluggish and maintained temporal response (Breitmeyer \& Ganz, 1976; Ingling, 1978).

Another suggestive result is the observation that TBE is abolished if the stimulus is in "hue substitution" (Bo- wen \& Nissen, 1979). With hue substitution, pulses of chromatic light are exchanged for an achromatic background of identical luminance. Such stimuli represent a pure chromatic stimulus; chromatic pulses that include a luminance increment do produce TBE. Since hue substitution is thought to "silence" the achromatic mechanism (Bowen, 1981), this result suggests that TBE is mediated by the "achromatic" or "luminance" channel of psychophysics (e.g., Guth, Massof, \& Benzschawel, 1980) but not by the "chromatie" channels. Since the "achromatic" and "chromatic" mechanisms of color vision psychophysics are often equated, respectively, with the "transient" and "sustained" mechanisms of spatial and temporal psychophysics (Ingling, 1978), all of the above effects confine the brightness enhancement effect to the achromatic or transient channel.

This hypothesis concerning the nature of TBE points up a further hypothesis, that individual differences between observers are based upon observers' "weighting" the chromatic and achromatic mechanisms differently. Type A observers rely largely on the output of achromatic (transient) channels, whereas Type $\mathrm{C}$ observers weight the chromatic (sustained) channels heavily. Type $\mathrm{C}$ observers may be thought of as using brightness information from the relatively "linear" chromatic channel, whereas the Type A observers depend on additional in- 
formation from the highly nonlinear achromatic channel. Type B observers utilize one class of mechanism under certain stimulus conditions and rely on the other mechanism under other conditions.

The channel weighting hypothesis predicts differences between the types of observers in any psychophysical task that can be interpreted as differentiating chromatic from achromatic mechanisms (see Bowen, 1981).

Drum (1980) has observed individual differences in the "spatial Broca-Sulzer effect" (Higgins \& Rinalducci, 1975), the fact that targets of intermediate size can appear brighter than larger or smaller targets of the same luminance. These differences can also be accounted for in the context of a "two-channel" model of brightness. A strong prediction (B. Drum, personal communication, 1984) of the weighting hypothesis is that Type $C$ observers, who exhibit weak temporal brightness enhancement, should show strong spatial brightness enhancement, assuming that the chromatic channels are preferentially sensitive to small targets (Ingling, 1978) and are thus responsible for spatial brightness enhancement (Drum, 1980). The reverse should be true for Type A observers.

If the underlying basis for individual differences is the weighting of achromatic and chromatic mechanisms, the weights adopted by an individual appear to be labile, based on the present results. Type $\mathrm{C}$ observers, with practice, can apparently learn to use information from the achromatic channel. Type B observers can shift between channels in response to changing stimulus conditions, weighting chromatic information heavily for simultaneous pulse onset conditions (in which the observer is faced with a difficult "peak" discrimination) and weighting achromatic information heavily for simultaneous pulse offset conditions (in which the short-pulse "peak" response can be compared to the "sustained" response to the comparison pulse).

The practice effects found in Experiment 3 clarify one ambiguity in a previous study (Bowen et al., 1982). We reported that different classes of observers showed no differences in sensitivity to sine-wave flicker modulation or to contrast sensitivity for drifting sine-wave gratings. Since these were threshold judgments, it is conceivable that differences in underlying visual neurophysiology or visual temporal processing could exist between observers at suprathreshold levels. But since mere practice would presumably not alter the neurophysiological substrate, the fact that observers can change with practive is best interpreted as a shift in either "channel weighting" or "perceptual criteria."

Finally, the existence of practice effects holds out the possibility that the classification of an observer will change fairly readily with perceptual training. If the "channel weighting"' hypothesis is correct, training could, in turn, modify visual function in any stimulus situation involving concurrent activation of chromatic and achromatic visual mechanisms.

\section{REFERENCES}

Berman, S. M., \& Stewart, A. L. (1978). Laterally induced impedance effects in vision. Journal of Mathematical Psychology, 18, 73-99.

BowEN, R. W. (1981). Latencies for chromatic and achromatic visual mechanisms. Vision Research, 21, 1457-1466.

Bowen, R. W., \& MARKell, K. A. (1980). Temporal brightness enhancement studied with a large sample of observers: Evidence for individual differences in brightness perception. Perception \& Psychophysics, 27, 465-476.

BOWEN, R. W., \& NISSEN, M. J. (1979). Luminance, not brightness, determines temporal brightness enhancement with chromatic stimuli. Journal of the Optical Society of America, 69, 581-584.

Bowen, R. W., \& POKORNy, J. (1978). Target edge sharpness and temporal brightness enhancement. Vision Research, 18, 1691-1695.

Bowen, R. W., Sekuler, R., Owsley, C. J., \& Markell, K. A. (1982). Individual differences in brightness perception. Perception \& Psychophysics, 30, 587-593.

BreItMEYER, B., \& GANZ, L. (1976). Implications of sustained and transient channels for theories of visual pattern masking, saccadic suppression and information processing. Psychological Review, 83, 1-36.

Broca, D., \& SUlzer, A. (1902). La sensation lumineuse en fonction du temps. Journal de Physiologie et de Pathologie Generale, 4, 632-640.

Drum, B. (1980). Relation of brightness to threshold for light-adapted and dark-adapted rods and cones: Effects of retinal eccentricity and target size. Perception, 9, 633-650.

Guth, S. L., Massof, R.W., \& Benzschawel, T. (1980). Vector model for normal and dichromatic color vision. Journal of the Optical Society of America, 70, 197-212.

HigGins, K.E., \& RiNALDUCCI, E.J. (1975). Suprathreshold intensityarea relationships: A spatial Broca-Sulzer effect. Vision Research, 15, 129-143.

INGLING, C. R. (1978). Luminance and opponent color contributions to visual detection and to temporal and spatial integration: Comment. Journal of the Optical Society of America, 68, 1143-1146.

KeLlY, D. H., \& SAVoIE, R. E. (1978). Theory of flicker and transient responses. III. An essential nonlinearity. Journal of the Optical Society of America, 68, 1481-1490.

Kitterle, F. L., \& Corwin, T. R. (1983). The effects of temporal waveform upon apparent contrast. Perception \& Psychophysics, 33, $72-74$.

Kitterle, F. L., \& Toney, H. C. (1983). The effects of temporal waveform upon temporal darkness enhancement. Perception \& Psychophysics, 33, 375-378.

Lupp, U., Hauske, G., \& Wolf, W. (1978). Different systems for the visual detection of high and low spatial frequencies. Photographic Science and Engineering, 22, 80-84.

OSAKA, N. (1982). Exponent of the Broca-Sulzer flash duration as a function of retinal eccentricity. Journal of the Optical Society of America, 72, 62-67.

WOODWORTH, R. S., \& SCHLOSBERG, H. (1954). Experimental psychology. New York: Holt, Rinehart and Winston.

\section{NOTES}

1. Berman and Stewart (1978) have presented a quantitative model of TBE. In their model, a single "net output function"' (response level as a function of time) can be used to predict nonmonotonic brightnessduration relations for a large class of possible weighted averages of the output function.

2. In the studies by Kitterle and Corwin (1983) and Kitterle and Toney (1983), there is a small, but consistent, effect of pulse offset. Ramping off an increment or decrement shifts the peak of the temporal enhancement effect to a longer pulse duration and reduces its magnitude. Thus, offset transients may also play a role in TBE. (See also Kelly and Savoie, 1978, and Bowen et al., 1982.) 